\title{
Increased Generation of Neuronal Progenitors after Ischemic Injury in the Aged Adult Human Forebrain
}

\author{
Jadranka Macas, Christian Nern, Karl H. Plate, and Stefan Momma \\ Institute of Neurology (Edinger Institute), University of Frankfurt, D-60528 Frankfurt, Germany
}

The adult human brain retains the capacity to generate new neurons in the hippocampal formation (Eriksson et al., 1998) and neuronal progenitor cells (NPCs) in the forebrain (Bernier et al., 2000), but to what extent it is capable of reacting to injuries, such as ischemia, is not known. We analyzed postmortem tissue from normal and pathological human brain tissue $(n=54)$ to study the cellular response to ischemic injury in the forebrain. We observed that cells expressing the NPC marker polysialylated neural adhesion cell molecule (PSANCAM) are continuously generated in the adult human subventricular zone (SVZ) and migrate along the olfactory tracts. These cells were not organized in migrating chains as in the adult rodent rostral migratory stream, and their number was lower in the olfactory tracts of brains from old (56-81 years of age) compared with young ( $29+36$ years of age) individuals. Moreover, we show that in brains of patients of advanced age ( $60-87$ years of age), ischemia led to an elevated number of Ki-67-positive cells in the ipsilateral SVZ without concomitant apoptotic cell death. Additionally, ischemia led to an increased number of PSA-NCAM-positive NPCs close to the lateral ventricular walls, compared with brains of comparable age without obvious neuropathologic changes. These results suggest that the adult human brain retains a capacity to respond to ischemic injuries and that this capacity is maintained even in old age.

Key words: ischemia; human; subventricular zone; neural progenitor cells; aging; neuropathology

\section{Introduction}

In the adult mammalian brain, new neurons are continuously added to the dentate gyrus of the hippocampus (Altman and Das, 1965; Cameron et al., 1993) and the olfactory bulb (Kaplan and Hinds, 1977; Luskin, 1993). The origin of this neurogenic activity and the locations where most neural stem or progenitor cells reside are the subgranular zone and periventricular area of the hippocampus and the subventricular zone (SVZ) of the forebrain (Gage, 2000). In an organ that was so far seen as highly static with little capacity for self-repair, these findings have raised hopes for future therapeutic prospects. Indeed, an increasing number of studies on rodents provide evidence that an injury response can be mounted from these neurogenic areas with an increased generation of neuronal progenitor cells (NPCs). Global ischemia leads to the proliferation of endogenous neural progenitors in the hippocampus (Liu et al., 1998; Nakatomi et al., 2002) and focal ischemia induces neural progenitors from the SVZ to proliferate and to migrate toward the lesion (Zhang et al., 2001; Arvidsson et al., 2002). Additionally, the injury response can be modulated by infusion of growth factors at appropriate time points (Nakatomi et al., 2002).

However, because most of the existing studies were conducted on rodent injury models and with respect to the reported differ-

\footnotetext{
Received May 16, 2006; accepted Nov. 12, 2006.

This work was supported by the Ludwig Edinger Foundation and a junior research grant from the Frankfurt University Medical School. We thank R. Cassidy and J. Frisén for comments on this manuscript, E. Dantas-Momma for proofreading and M. Melis for help with human tissue.

Correspondence should be addressed to Dr. Stefan Momma, Edinger Institute, University of Frankfurt, Deutschordenstrasse 46, D-60528 Frankfurt, Germany. E-mail: stefan.momma@kgu.de.

DOI:10.1523/JNEUROSCI.4667-06.2006

Copyright $\odot 2006$ Society for Neuroscience $\quad$ 0270-6474/06/2613114-06\$15.00/0
}

ences between primates and rodents, their applicability to humans has to be treated with caution (Rakic, 2004; Sanai et al., 2004). Although there is evidence for an increased generation of hippocampal NPCs in chronic neurodegenerative diseases in humans (Curtis et al., 2003; Jin et al., 2004), no data are available about the adult human forebrain reaction to ischemic injury. Therefore, in the present study, we wanted to address whether this type of injury can elicit a response from endogenous neural stem or progenitor cells. We analyzed cell proliferation and expression of neuronal progenitor antigens in the SVZ of adult human brains from postmortem cases of injured and nonpathological brains. Because ischemic injuries typically occur more frequently in older individuals, we also assessed the extent of NPC generation and migration along the olfactory tracts depending on age.

\section{Materials and Methods}

Human specimens. Autopsied human brains were obtained within $24 \mathrm{~h}$ of death from the Department of Pathology of the University Hospital Frankfurt or as tissue blocks embedded in paraffin from the Edinger Institute archive. As control for injury studies, only brains that displayed no neuropathological changes from individuals without neurological symptoms were used. We excluded brains from individuals that received chemotherapy or were irradiated in the course of tumor therapy. Brains were cut coronally at the corpora mammilaria, and the lateral ventricular walls, optical tracts, and bulbs were dissected out and placed in paraformaldehyde (4\%), pH 7.4, for at least $24 \mathrm{~h}$. All work involving human tissue was approved by the ethical committee of the University Hospital Frankfurt.

Histology. Blocks of tissue were embedded in paraffin after fixation and 3-4 $\mu \mathrm{m}$ sections were cut on a microtome (SM2000R; Leica, Nussloch, Germany). The following antibodies were used: mouse anti- $\beta$-III- 

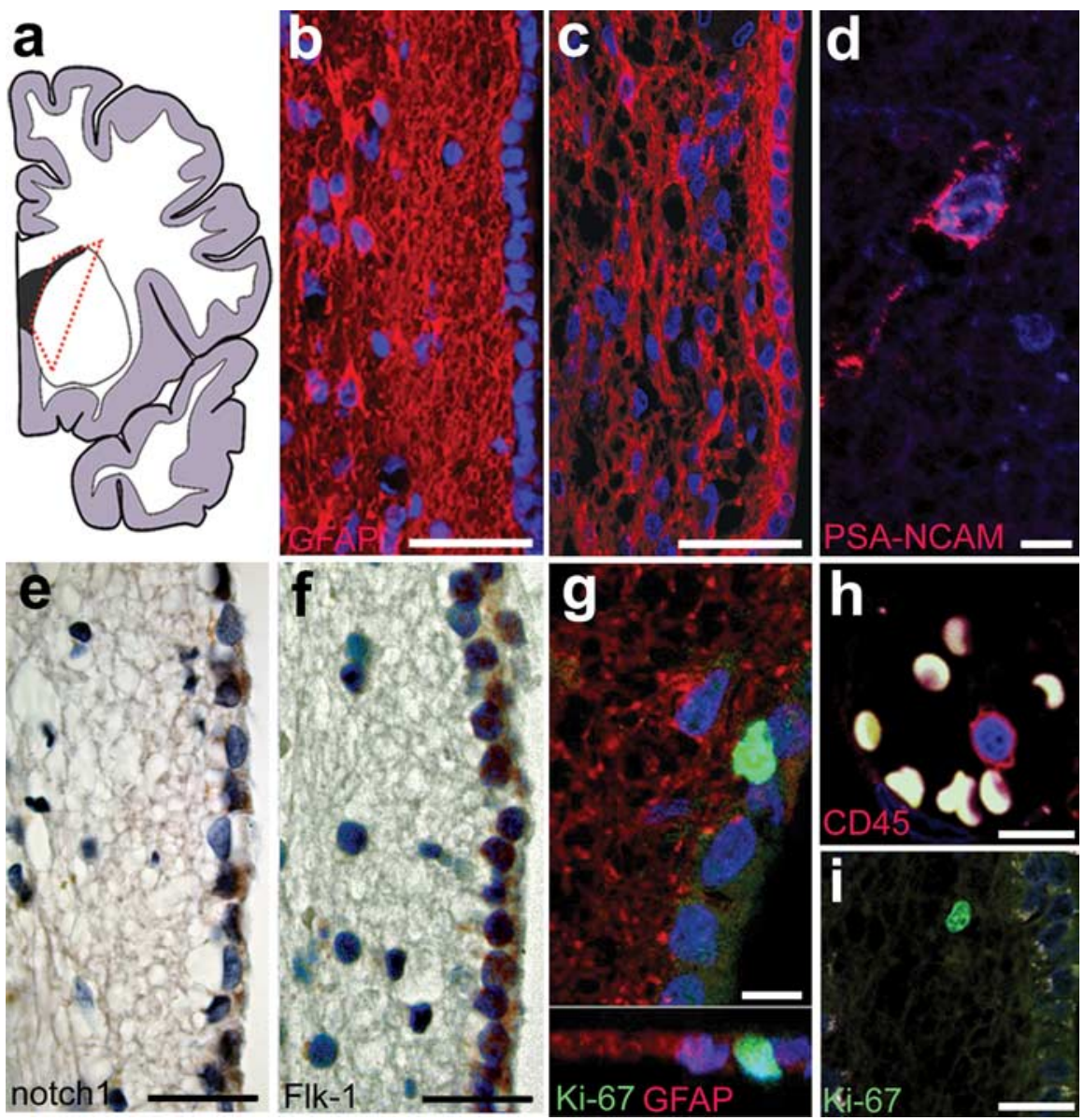

Figure 1. Cellular architecture and proliferative capacity of the adult human SVZ. $\boldsymbol{a}$, Schematic overview of a human brain hemisphere with the dotted red line indicating the area of analysis. $\boldsymbol{b}, \boldsymbol{c}$, Representative histology of the SVZ with a gap between the ependymal layer and astrocytic ribbon in the medial part $(\boldsymbol{b})$ and with the astrocytic ribbon being continuous from the ependymal layer to the brain parenchyma in the dorsal and ventral areas (c). $\boldsymbol{d}$, Occasionally, PSA-NCAM-positive cells can be detected close to the ependyma or in the astrocytic ribbon and beyond. $\boldsymbol{e}, \boldsymbol{f}$, Molecules that were shown to regulate neurogenesis such as notch1 (e) and Flk-1/VEGFR-2 $(\boldsymbol{f})$ are expressed in the ependymal cell layer. $\boldsymbol{g}$, Proliferating Ki-67-positive cells are often closely associated with the ependymal layer. $\boldsymbol{h}, \boldsymbol{i}$, Lack of costaining with pan-hematopoietic markers such as CD45 (h) rules out a non-neural origin of the proliferating Ki-67-positive cells (i). Scale bars: $\boldsymbol{b}, \boldsymbol{c}, 50 \mu \mathrm{m} ; \boldsymbol{d}, \boldsymbol{g}, \boldsymbol{h}, 10 \mu \mathrm{m} ; \boldsymbol{e}, \boldsymbol{f}, 25 \mu \mathrm{m} ; \boldsymbol{i}, 20 \mu \mathrm{m}$.

tubulin (clone Tuj1 1:500; Covance), monoclonal mouse anti-polysialic acid neural cell adhesion molecule (PSA-NCAM, clone 2-2B, 1:2000; Millipore, Temecula, CA), monoclonal mouse anti-human notch1 (1: 2000, Ab-1, clone A6; Biocarta Europe, Hamburg, Germany), polyclonal rabbit anti- vascular endothelial growth factor -receptor-2 (VEGFR2)/ fetal liver kinase receptor 1 (Flk-1) (1:100; Zytomed, Berlin, Germany), monoclonal mouse anti-glial fibrillary acidic protein (GFAP, 1:500; Millipore), polyclonal rabbit anti-GFAP (1:1000; Dako, Carpinteria, CA), monoclonal mouse anti-human CD45/leukocyte common antigen (1:50, clones 2B11+PD7/26; Dako), monoclonal mouse anti-human CD68 (clone PG-M1, 1:100; Dako), monoclonal mouse anti-human CD34 (clone QBEND10, 1:100; Immunotech, Marseille, France), polyclonal rabbit anti-human $\mathrm{S} 100 \beta$ (1:100; Dako), monoclonal mouse antihuman nestin (1:200; Millipore), polyclonal rabbit anti-Mash1 (1:100; Millipore), polyclonal rabbit anti-Musashi (1:100; Millipore), and monoclonal mouse anti-human Ki-67 (clone MIB-1, 1:50; Dako). For detection of Ki-67, antigen retrieval was performed by heating the tissue in a $10 \mathrm{~mm}$ citric acid buffer, $\mathrm{pH}$ 6.0. For peroxidase immunohistochemistry, antibody staining was visualized using the StrAviGen Multilink-RKit (BioGenex, San Ramon, CA) with 3-amino-9-ethyl-carbazole in $\mathrm{N}, \mathrm{N}$-dimethylformamide as a chromogen (AEC; BioGenex). Images for light microscopy were taken on an Olympus (Tokyo, Japan) BX51 microscope using the Analysis Software package (Soft Image Systems, Hamburg, Germany). Antibody staining was visualized with the following secondary antibodies: goat anti-mouse IgG Alexa Fluor 568 and 488, goat anti-rabbit IgG Alexa 568 and 488 (all from Invitrogen, Eugene, $\mathrm{OR}$ ) and goat anti-mouse IgM Cy3 (Millipore). All fluorescence images were recorded on a Leica TCS SP confocal microscope.

Quantitation. For a detailed description of the quantitation for all case studied see supplemental material, available at www.jneurosci.org.

TUNEL. Detection of the DNA fragmentation characteristic of apoptosis was performed using a terminal deoxynucleotidyl transferasemediated biotinylated UTP nick end labeling (TUNEL) assay based on the Apop Tag Plus fluorescein In Situ Apoptosis Detection Kit (Millipore) according to instructions of the manufacturer.

\section{Results}

Morphology of the adult human SVZ

As a first step, we examined the morphology of the lateral ventricular walls of the anterior horn (Fig. 1a) along the dorsoventral and anterior-posterior axis of adult human brains without any apparent neuropathological changes (for a complete list of autopsy cases see supplemental Table 1, available at www.jneurosci.org as supplemental material). The general morphology of the middle part of the lateral walls of the lateral ventricles was a single ependymal cell layer lining the ventricular wall next to a gap, followed by a ribbon of GFAP-positive astrocytes as described previously (Sanai et al., 2004; QuinonesHinojosa et al., 2006) (Fig. 1b), whereas in the dorsal and ventral parts there was a continuum of cells starting from just beneath the ependymal layer (Fig. $1 c)(n=$ 37). PSA-NCAM is expressed by migrating neuroblasts in the adult rostral migratory stream and SVZ in rodents (Doetsch and Alvarez-Buylla, 1996) as well as in human NPCs (Bernier et al., 2000). We detected occasional PSA-NCAMpositive cells close to the lateral ventricular wall as reported previously (Fig. 1d) (Bedard and Parent, 2004; Sanai et al., 2004), suggesting an ongoing production of NPCs, but these cells were not organized as migrating clusters as in the rodent brain. Interestingly, molecules that are known to play a role in the regulation of stem cells and neurogenesis in the rodent brain, such as notch1 (Gaiano and Fishell, 2002) (Fig. 1e) and VEGFR-2/Flk-1 (Schanzer et al., 2004) (Fig. 1f) were expressed in the nearby ependymal cell layer. Using the cell cycle marker Ki-67, we found occasional proliferating cells in the SVZ (Fig. $1 g$ ). Many of these cells were located directly underneath the ependymal layer, but not in the ependymal layer itself, similar to observations of proliferating cells in the SVZ of the adult macaque monkey brain (Kornack and Rakic, 2001). Approximately thirty percent of the Ki-67positive cells were located in the astrocytic ribbon and stained for GFAP $(n=3 ; 33.7 \pm 5.51 \% \mathrm{SD})$ and some were TuJ1-positive $(n=3 ; 2.2 \pm 0.98 \% \mathrm{SD})$. However, the majority of these cells did not costain with any other neural marker used ( $100 \beta$, nestin, musashi, mash1). We could not establish any difference in the distribution of labeled cells analyzed, depending on the dorso- 

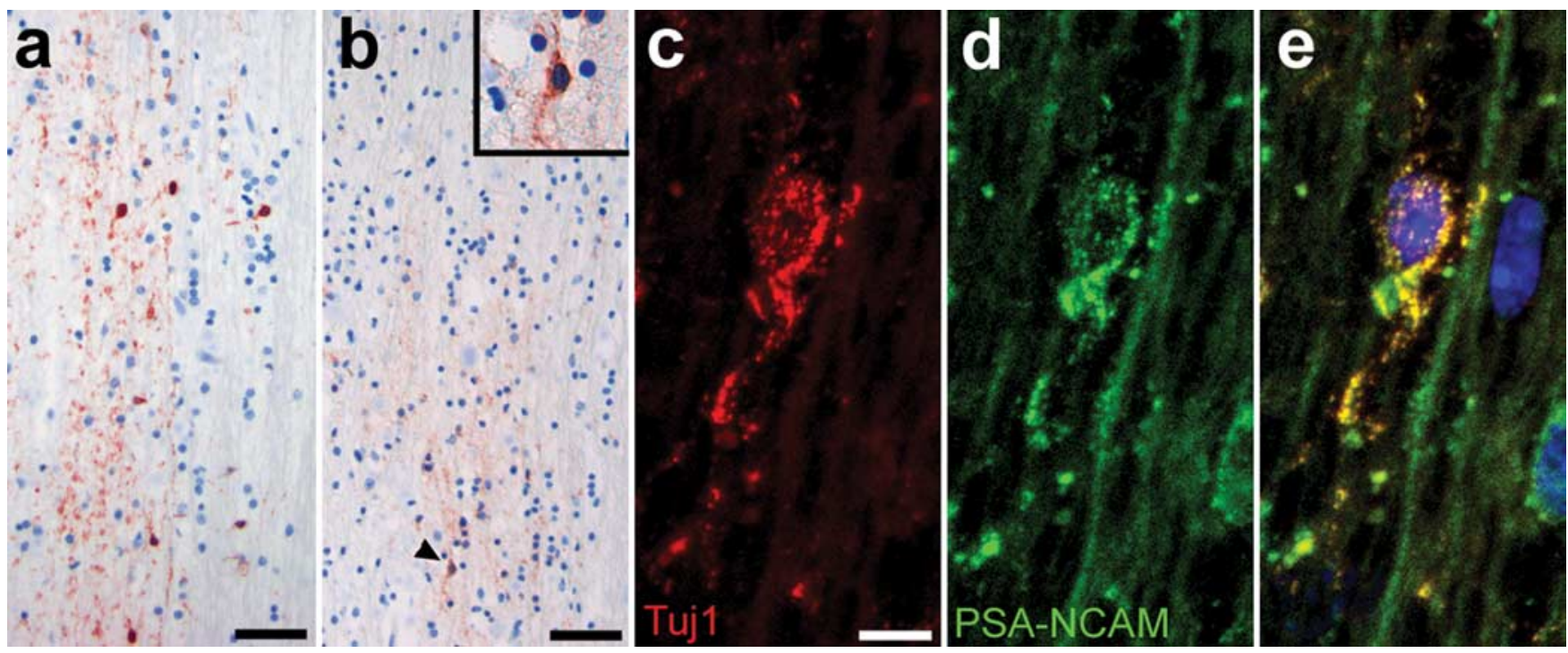

Figure 2. Decrease in the production of NPCs migrating along the olfactory tract with age. $\boldsymbol{a}, \boldsymbol{b}, 0$ lfactory tracts of younger individuals ( $\boldsymbol{a}$ ) show a much higher number of PSA-NCAM-positive cells than those of old patients $(\boldsymbol{b})$. c, PSA-NCAM-positive cells have an elongated morphology ( $\boldsymbol{b}, \boldsymbol{d}$, inset) and costain with the marker TuJ1. $\boldsymbol{e}$, Merged image with T0T0-3 iodide-labeled nuclei. Scale bars: $\boldsymbol{a}, \boldsymbol{b}, 25 \mu \mathrm{m} ; \boldsymbol{c}, 10 \mu \mathrm{m}$

ventral position along the ventricular walls. To exclude cells of non-neuroepithelial origin in our analysis, we identified resident microglia and infiltrating cells of hematopoietic origin with the markers CD45, CD34, and CD68 in adjacent sections from all brains analyzed. All brains that were included in our analysis showed no colabeling of any of these markers with Ki-67 in the region of interest, except in blood vessels (example of a CD45-positive leukocyte in a blood vessel and Ki-67 in the SVZ) (Fig. 1h,i).

\section{The number of NPCs migrating along the olfactory tracts decreases with age}

Ischemic injuries are more common in older patients although neurogenesis decreases in aged animals (Kuhn et al., 1996; Tropepe et al., 1997). Therefore, we wanted to test whether we could find evidence of a reduced generation of NPCs in the aged human brain. For that reason we compared the number of PSANCAM-positive cells in sections of the olfactory tracts from young individuals (Fig. $2 a$ ) with those of individuals of advanced age (Fig. $2 b$ ). PSA-NCAM-labeled cells were fewer in the olfactory tracts of old patients $(n=3 ; 56$ years of age, $40 \pm 16$ cells $/ 10$ $\mathrm{mm}^{2}$; 58 years of age, $23.3 \pm 1.5$ cells $/ 10 \mathrm{~mm}^{2} ; 81$ years of age, $20.7 \pm 2.5$ cells $/ 10 \mathrm{~mm}^{2}$; all means $\pm \mathrm{SD}$ ) as compared with those of young individuals $(n=2 ; 29$ years of age, $175.3 \pm 25.5$ cells $/ 10$ $\mathrm{mm}^{2}$; 36 years of age, $210.7 \pm 37.5$ cells $/ 10 \mathrm{~mm}^{2}$; all means \pm $\mathrm{SD})$. In all cases, most, but not all PSA-NCAM-positive cells in the olfactory tract colabeled with the early neuronal marker TuJ1 (Fig. 2c-e). However, PSA-NCAM-positive cells were not organized as migrating chains, which is a hallmark of the rodent rostral migratory stream, but rather remained as individual cells. If the occurrence of cells bearing markers of migrating NPCs reflects actual ongoing neurogenesis, our data indicates that the generation of new neurons in the olfactory bulb is downregulated with age.

\section{Ischemic injuries lead to an increase in cell proliferation and} numbers of NPCs in the SVZ

Ischemic injuries are caused by disruption of cerebral blood flow often resulting in neurological deficits with limited prospects of improvement. Therefore, we analyzed brains of patients with ischemic damage $(n=3)$ that died $5 \mathrm{~d}, 8 \mathrm{~d}$, and 4 months after injury (for details of quantified cases, see supplemental Table 2, available at www.jneurosci.org as supplemental material). Figure $3 a$ shows a schematic drawing of a brain of one patient (87 years of age) that died $5 \mathrm{~d}$ after a massive hemilateral ischemia caused by thrombosis of the right middle cerebral artery. Because the reaction to ischemic damage is much more pronounced in the hemisphere ipsilateral to the insult (Arvidsson et al., 2002), this particular case allowed us to compare the two hemispheres with the contralateral side serving as an internal reference. For quantitation, we divided the SVZ into two zones (Fig. 3b). Quantitation of Ki-67-positive cells in the area close to the lateral wall including the astrocytic ribbon of the lateral ventricle (Fig. $3 b$, zone I) revealed an increase in the number of labeled cells compared with uninjured control brains $(n=5)$ (Fig. $3 c, d, j)$. We observed no increase of Ki-67-positive cells in the brain of a patient that died 4 months after the injury (Fig. 3j, apallic syndrome, 60 years of age). With respect to the brain for which we had the contralateral hemisphere as a reference (stroke, 87 years of age), the ipsilateral side to the injury contained much higher levels of Ki-67-positive cells (Fig. 3j) and a twofold increase in PSANCAM-positive NPCs in zone II, but no difference in zone I compared with the contralateral side (Fig. $3 e, f, k$ ). The number of PSA-NCAM-positive cells was also significantly increased in the case of a patient with apallic syndrome (Fig. $3 k$ ). This increase was higher in zone II than zone I, pointing to at least a limited migratory capacity of neuronal progenitor cells toward the parenchyma. Interestingly, the number of Ki-67 and PSA-NCAMpositive cells did not differ significantly (tested by one-way ANOVA) among the control brains, despite their wide age range (19-87 years of age). In any case, levels of proliferation and of NPCs in the developing human ventricular zone (week 26 of pregnancy) (Fig. $3 g, h$ ) are much higher compared with any adult brain. Cells undergoing apoptotic cell death can express Ki-67 (Yang et al., 2001), but the increase of this marker was not concurrent with an increase of TUNEL-positive apoptotic cells in the SVZ compared with the contralateral side to the injury or to control brains (data not shown). In the rodent brain, at least 


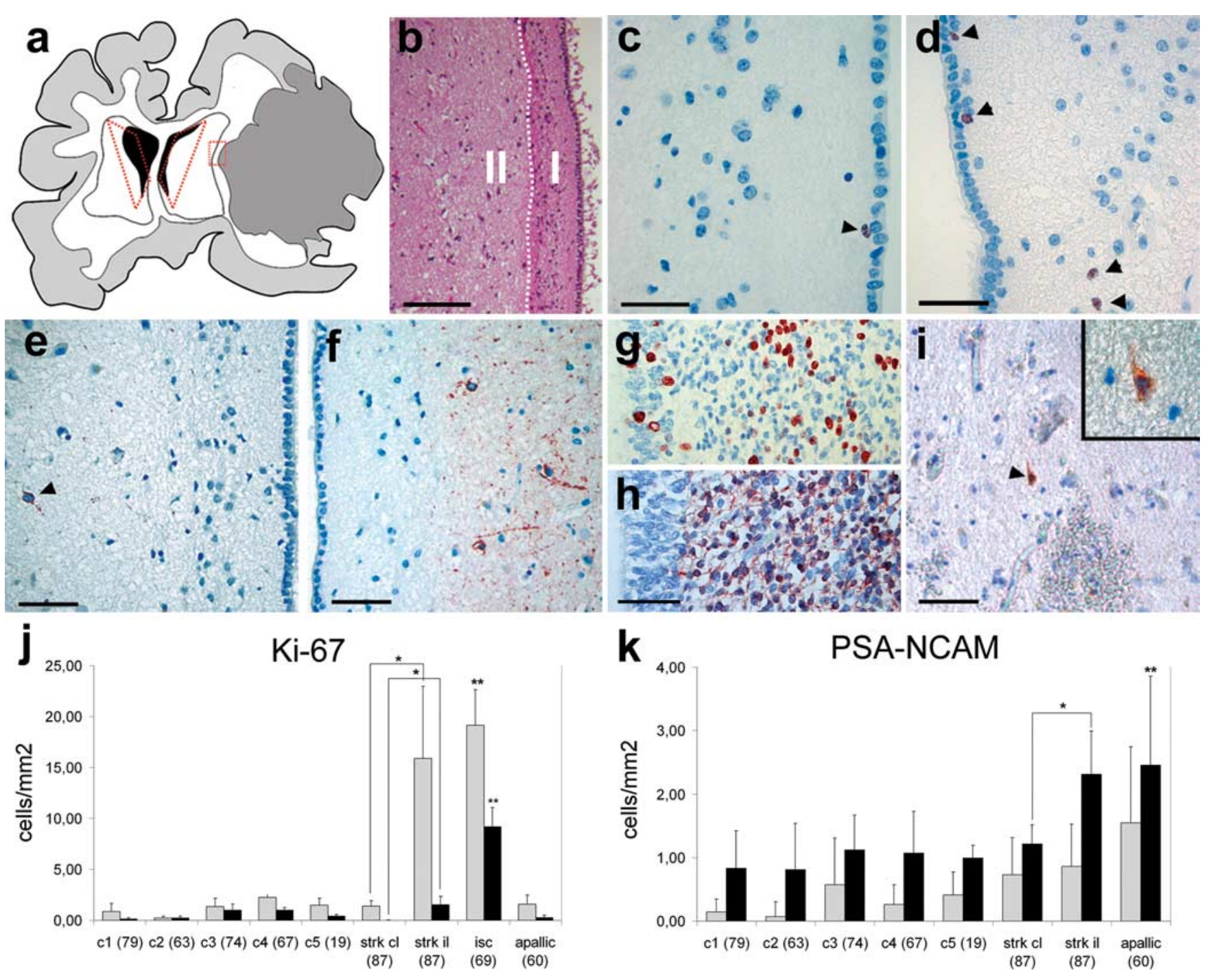

Figure 3. Increase in cell proliferation and production of PSA-NCAM-positive NPCs in ischemic injuries. $\boldsymbol{a}$, Schematic drawing of a human brain from a patient that died $5 \mathrm{~d}$ postischemia with a large area of necrotic tissue in the right hemisphere (area shaded in gray). The areas that were analyzed by immunohistochemistry are delineated by red dotted lines. $\boldsymbol{b}$, For quantitation, the subventricular area was divided into two zones: zone I was defined as the area from the ependymal layer to the adjacent brain parenchyma including the astrocytic ribbon (white dotted line); zone Il encompasses the tissue from the astrocytic ribbon to deeper layers of the brain parenchyma. $\boldsymbol{c}, \boldsymbol{d}$, In the ipsilateral side to the injury, the number of proliferating cells close to the lateral ventricular walls increased ( $\boldsymbol{d}$ ) compared with the contralateral side (c). Ki-67-positive cells are indicated with arrowheads. $\boldsymbol{e}, \boldsymbol{f}$, Likewise, the production of PSA-NCAM-positive NPCs is lower in contralateral (e) compared with the ipsilateral side $(\boldsymbol{f}) . \boldsymbol{g}, \boldsymbol{h}$, Proliferation $(\boldsymbol{g})$ and the number of PSA-NCAM-positive NPCs $(\boldsymbol{h})$ is much higher in the ventricular zone of the late-developing compared with the adult human brain. $\boldsymbol{i}$, PSA-NCAM-positive cells can be detected only rarely at the penumbra of the stroke area (area indicated by red dotted rectangle in $\boldsymbol{a}$ ). $\boldsymbol{j}, \boldsymbol{k}$, Quantitation of Ki-67- (j) and PSA-NCAM-positive $(\boldsymbol{k})$ cells per millimeter squared in zone I and II; ipsilateral and contralateral sides were compared with each other for both zones and each ischemic case with every control. Error bars indicate $\pm S D$. Statistical significance was calculated by Student's unpaired $t$ test; $j_{1}{ }^{*} p<0.005,{ }^{* *} p<0.0005 ; \boldsymbol{k}_{,}{ }^{*} p<0.05,{ }^{* *} p<0.005$. Controls were not significantly different from each other, assessed by one-way ANOVA. Scale bars: $\boldsymbol{b}, 100 \mu \mathrm{m} ; \boldsymbol{c}, \boldsymbol{d}, 20 \mu \mathrm{m} ; \boldsymbol{e}, \boldsymbol{f}, \boldsymbol{h}, \boldsymbol{i}, 50 \mu \mathrm{m}$.

some cells of the SVZ are redirected toward the site of injury, where they can generate neurons or glia (Arvidsson et al., 2002). In contrast, we detected $<0.15$ cells $/ \mathrm{mm}^{2}$ PSA-NCAM-positive cells in sections taken from the border area of the ischemic injury (Fig. 3a,I, red dotted rectangle). This could be attributable to a more restricted migratory capacity of human cells or that the time frame of $5 \mathrm{~d}$ after injury until death was not sufficient to allow for more extensive generation and migration of NPCs over such distances.

Next, we asked whether other structures in the brain outside the SVZ could be involved in the generation of NPCs. Therefore we examined cortical regions adjacent to the injured tissue for the possibility of a local source for PSA-NCAM-positive cells and compared this to the equivalent region in the noninjured hemisphere. In 10 sections analyzed from cortical areas in both hemi- spheres, we found comparable low levels of PSA-NCAM-positive cells (total area of $>8.6 \mathrm{~cm}^{2}$ for each hemisphere; $0.027 \pm 0.034$ cells $/ \mathrm{mm}^{2}$ in the injured and $0.025 \pm 0.029$ cells $/ \mathrm{mm}^{2}$ in the noninjured hemisphere; means $\pm \mathrm{SD}$ ). We conclude that a local pool of NPCs may exist, but that their absolute number is too low to assess a reaction to injury.

\section{Discussion}

Our results show that the adult human brain has the capacity to react to ischemic injuries with an increased generation of NPCs in the SVZ. Although we observed a decline in the number of NPCs migrating along the olfactory tracts in older patients, the injury reactions to ischemia occurred despite the advanced age of the affected individuals.

The cellular architecture of the human SVZ differs from the 
homologous areas in brains of lower vertebrates, notably by the absence of rapidly dividing precursor cells and a very low number of NPCs. However, as in rodents, some constituents of the mammalian neurogenic niche, such as VEGFR-2 and Notch1, are expressed in the ependymal cell layer of the human SVZ. Although it has been reported that in the human SVZ the ependymal layer is separated from underlying cells by a cell free gap (Sanai et al., 2004), we found this particular organization predominantly in the medial part of the lateral walls of the lateral ventricles. In contrast, in the dorsal and ventral parts, cells form a continuum from the ependyma to the striatum and Ki-67-expressing cells are frequently located underneath, but not within the ependymal layer, although we could not establish a significant difference in the number of Ki-67 or PSA-NCAM-labeled cells depending on the dorsoventral position.

In line with a continuous generation of NPCs in the SVZ, we observed cells expressing markers of migrating NPCs in adult human olfactory tracts. Their number decreased with age, consistent with observations of decreasing neurogenesis in old animals (Tropepe et al., 1997). These age differences may account for the different observations from previous studies regarding the ongoing generation of NPCs and their migration toward the olfactory bulb (Bernier et al., 2000; Bedard and Parent, 2004; Sanai et al., 2004). Interestingly, the number of PSA-NCAM- and Ki67-positive cells in the SVZ among the control cases did not differ significantly despite the wide age range from 19-87 years. A possible explanation could be that the age-related difference in the number of NPCs in the olfactory tracts is attributable to selective cell death when cells have migrated out of the SVZ. In any case, even in young individuals, migrating NPCs are not organized in dense clusters that are characteristic for the rostral migratory stream of NPCs in the rodent forebrain. Thus, the human cells appear to migrate as individual cells, as suggested previously (Sanai et al., 2004). Expression of markers for migrating NPCs per se is no proof for actual migration and neurogenesis. Ultimately, evidence for adult human forebrain neurogenesis would have to come from studies such as those using bromodeoxyuridine-labeled tissue as for the demonstration of new neurons in the hippocampus (Eriksson et al., 1998).

We observed an increase in the number of PSA-NCAMpositive cells in the SVZ in brains with ischemic lesions and a strong upregulation of proliferation, measured by detection of $\mathrm{Ki}-67$, in brains of patients that died just few days after ischemia. Ischemic lesions to the hippocampus or forebrain have been shown to enhance the proliferation and generation of NPCs in rodents and in macaque monkeys (Tonchev et al., 2003, 2005). Although the absolute number of newly generated NPCs is low, recent evidence suggests that the continuing addition of small numbers of new neurons to the injured striatum over long periods can add up to a significant amount of cells (Thored et al., 2006). The increased number of PSA-NCAM-positive cells in zone II, together with elevated levels of Ki-67-positive cells in zone I after ischemia suggests an, at least limited, ability of neuronal progenitor cells to migrate into the brain parenchyma. Although we found no convincing evidence for the migration of newly generated cells into the lesioned area, this may be because of the insufficient time passed after injury. However, a study using a global ischemia model in the adult macaque monkey (Tonchev et al., 2005) showed that cells did not deviate from their normal migratory pathway toward the olfactory bulb, pointing to a general difference in neural progenitor cell behavior in primates compared with rodents. The number of PSA-NCAM cells in the SVZ of the apallic patient that died 6 months after ischemia is increased in the absence of elevated levels of Ki-67. This would be consistent with the interpretation that the increase in cell proliferation and NPC production after injury is transitory, and that the ability of NPCs to migrate out of the SVZ is very slow. Ultimately, the issue about the migratory capacity of neuronal progenitor cells in the human brain would have to be resolved by the analysis of ischemic brains of patients that died a long time after an injury.

NPCs can be isolated from the adult human subcortical white matter (Nunes et al., 2003). These cells may react to injury by locally contributing to the generation of new neurons. In contrast, we could not detect higher numbers of PSA-NCAMpositive cells close to the injury, or numbers that differed substantially from controls for cortical areas adjacent to the injury. Therefore we conclude that a local injury response is absent, not substantial enough in the cases studied, or not detectable with our methods.

Our study shows that endogenous neural precursor cells in the adult human brain react to ischemic injuries and that this reaction is maintained even in old age. This work emphasizes the importance of additional studies on the role and potential of endogenous neural precursor cells in the adult human brain.

\section{References}

Altman J, Das GD (1965) Autoradiographic and histological evidence of postnatal hippocampal neurogenesis in rats. J Comp Neurol 124:319-335.

Arvidsson A, Collin T, Kirik D, Kokaia Z, Lindvall O (2002) Neuronal replacement from endogenous precursors in the adult brain after stroke. Nat Med 5:5.

Bedard A, Parent A (2004) Evidence of newly generated neurons in the human olfactory bulb. Brain Res Dev Brain Res 151:159-168.

Bernier PJ, Vinet J, Cossette M, Parent A (2000) Characterization of the subventricular zone of the adult human brain: evidence for the involvement of Bcl-2. Neurosci Res 37:67-78.

Cameron HA, Woolley CS, McEwen BS, Gould E (1993) Differentiation of newly born neurons and glia in the dentate gyrus of the adult rat. Neuroscience 56:337-344.

Curtis MA, Penney EB, Pearson AG, van Roon-Mom WM, Butterworth NJ, Dragunow M, Connor B, Faull RL (2003) Increased cell proliferation and neurogenesis in the adult human Huntington's disease brain. Proc Natl Acad Sci USA 100:9023-9027.

Doetsch F, Alvarez-Buylla A (1996) Network of tangential pathways for neuronal migration in adult mammalian brain. Proc Natl Acad Sci USA 93:14895-14900.

Eriksson PS, Perfilieva E, Bjork-Eriksson T, Alborn AM, Nordborg C, Peterson DA, Gage FH (1998) Neurogenesis in the adult human hippocampus. Nat Med 4:1313-1317.

Gage FH (2000) Mammalian neural stem cells. Science 287:1433-1438.

Gaiano N, Fishell G (2002) The role of notch in promoting glial and neural stem cell fates. Annu Rev Neurosci 25:471-490.

Jin K, Peel AL, Mao XO, Xie L, Cottrell BA, Henshall DC, Greenberg DA (2004) Increased hippocampal neurogenesis in Alzheimer's disease. Proc Natl Acad Sci USA 101:343-347.

Kaplan MS, Hinds JW (1977) Neurogenesis in the adult rat: electron microscopic analysis of light radioautographs. Science 197:1092-1094.

Kornack DR, Rakic P (2001) The generation, migration, and differentiation of olfactory neurons in the adult primate brain. Proc Natl Acad Sci USA 98:4752-4757.

Kuhn HG, Dickinson-Anson H, Gage FH (1996) Neurogenesis in the dentate gyrus of the adult rat: age-related decrease of neuronal progenitor proliferation. J Neurosci 16:2027-2033.

Liu J, Solway K, Messing RO, Sharp FR (1998) Increased neurogenesis in the dentate gyrus after transient global ischemia in gerbils. J Neurosci 18:7768-7778.

Luskin MB (1993) Restricted proliferation and migration of postnatally generated neurons derived from the forebrain subventricular zone. Neuron 11:173-189.

Nakatomi H, Kuriu T, Okabe S, Yamamoto S, Hatano O, Kawahara N, 
Tamura A, Kirino T, Nakafuku M (2002) Regeneration of hippocampal pyramidal neurons after ischemic brain injury by recruitment of endogenous neural progenitors. Cell 110:429-441.

Nunes MC, Roy NS, Keyoung HM, Goodman RR, McKhann II G, Jiang L, Kang J, Nedergaard M, Goldman SA (2003) Identification and isolation of multipotential neural progenitor cells from the subcortical white matter of the adult human brain. Nat Med 9:439-447.

Quinones-Hinojosa A, Sanai N, Soriano-Navarro M, Gonzalez-Perez O, Mirzadeh Z, Gil-Perotin S, Romero-Rodriguez R, Berger MS, GarciaVerdugo JM, Alvarez-Buylla A (2006) Cellular composition and cytoarchitecture of the adult human subventricular zone: a niche of neural stem cells. J Comp Neurol 494:415-434.

Rakic P (2004) Neuroscience: immigration denied. Nature 427:685-686.

Sanai N, Tramontin AD, Quinones-Hinojosa A, Barbaro NM, Gupta N, Kunwar S, Lawton MT, McDermott MW, Parsa AT, Manuel-Garcia Verdugo J, Berger MS, Alvarez-Buylla A (2004) Unique astrocyte ribbon in adult human brain contains neural stem cells but lacks chain migration. Nature 427:740-744.

Schanzer A, Wachs FP, Wilhelm D, Acker T, Cooper-Kuhn C, Beck H, Winkler J, Aigner L, Plate KH, Kuhn HG (2004) Direct stimulation of adult neural stem cells in vitro and neurogenesis in vivo by vascular endothelial growth factor. Brain Pathol 14:237-248.

Thored P, Arvidsson A, Cacci E, Ahlenius H, Kallur T, Darsalia V, Ekdahl CT, Kokaia Z, Lindvall O (2006) Persistent production of neurons from adult brain stem cells during recovery after stroke. Stem Cells 24:739-747.

Tonchev AB, Yamashima T, Zhao L, Okano HJ, Okano H (2003) Proliferation of neural and neuronal progenitors after global brain ischemia in young adult macaque monkeys. Mol Cell Neurosci 23:292-301.

Tonchev AB, Yamashima T, Sawamoto K, Okano H (2005) Enhanced proliferation of progenitor cells in the subventricular zone and limited neuronal production in the striatum and neocortex of adult macaque monkeys after global cerebral ischemia. J Neurosci Res 81:776-788.

Tropepe V, Craig CG, Morshead CM, van der Kooy D (1997) Transforming growth factor- $\alpha$ null and senescent mice show decreased neural progenitor cell proliferation in the forebrain subependyma. J Neurosci 17:7850-7859.

Yang Y, Geldmacher DS, Herrup K (2001) DNA replication precedes neuronal cell death in Alzheimer's disease. J Neurosci 21:2661-2668.

Zhang RL, Zhang ZG, Zhang L, Chopp M (2001) Proliferation and differentiation of progenitor cells in the cortex and the subventricular zone in the adult rat after focal cerebral ischemia. Neuroscience 105:33-41. 